\title{
The Novel Gravitational Mass Weighted PCA Technique for Feature Extraction in Hyperspectral Data Classification
}

\author{
Nandhini K, Porkodi R
}

\begin{abstract}
Recent advancements in the imaging spectrometer collect both spatial and spectral information which creates a huge dimensionality. The heavy spectral information creates to build a classifier for discerning between the materials in the scene. The minimum number of training labels always in an exchange between the spectral information and the performance is called the Hughes effect. Also the redundant of spectral information and noisy data presents in the hyperspectral scene. The above issues are overcome using feature extraction and feature selection methods which play a major role in the reduction of dimensionality. This paper proposes the novel fusion Gravitational Mass Weighted Principal Component Analysis (GMWPCA) techniques for hyperspectral data dimensionality. Also, this paper presents the deep insight about the feature extraction techniques in hyperspectral data of both supervised and unsupervised learning methods and experimental analysis in AVIRIS Indian Pines hyperspectral dataset by employing PCA, Probability PCA, LDA, and proposed techniques. The $93.63 \%$ high accuracy achieved by using a novel proposed method.
\end{abstract}

Index Terms: Feature Extraction, Gravitational search algorithm, Gravitational weighted Mass PCA, Hyperspectral data, PCA

\section{INTRODUCTION}

The hundreds of continuous adjacent bands are generated as a hyperspectral (HS) cube. The less number of known samples only available with the large dimensional HS cube decreases the classification accuracy and increases the computational complexity. There are two fundamental techniques are often involved in the reduction of dimensionality are feature selection (FS) and feature extraction (FE) techniques. FS methods investigate the highly informative bands from the HS data and eliminate the other bands. The high dimensionality HS data are projected to low dimensionality without missing of any information is referred to as feature extraction. In FS techniques there might be a chance of missing the potential information which often leads to an effect in performance. Genetic algorithm, information theoretic approaches, global and local structure preservation framework and representation techniques are some of the feature selection methods. Basically in HS classification, the FE techniques are partitioned into different types: unsupervised, supervised and semi-supervised. An unsupervised FE method doesn't require known labels and also the class discrimination information. ICA, PCA, and MNF are often used in HS data as unsupervised feature extraction techniques [1].

Revised Version Manuscript Received on Jun 20, 2019.

Nandhini K, Department of Computer Science, Bharathiar University, Coimbatore, Tamilnadu, India.

Porkodi R, Department of Computer Science, Bharathiar University, Coimbatore, Tamilnadu, India.
Over the decades, supervised and unsupervised techniques are widely used in the extraction of the features of HS data Linear Discriminant Analysis (LDA), Generalized DA, MMLDA, NWFE, etc. are some of the supervised feature extraction techniques often been in comparison with other techniques and also widely used methods [1].

The research paper directed towards on the background study and analysis of supervised and unsupervised FE techniques. The remaining of the paper crafted as follows: Chapter II discusses the unsupervised feature extraction techniques in HS data. Chapter III discusses the supervised feature extraction techniques in HS data and Chapter IV discusses the generic methodology framework and Chapter V concluded the study and analysis along with future work.

\section{UNSUPERVISED FEATURE EXTRACTION TECHNIQUES WITH REFERENCE TO HS DATA}

The chapter offers an overview of the HS data feature extraction and in-depth analysis of the PCA and ICA techniques in the forthcoming sections.

\section{A. Principal Component Analysis (PCA)}

Karl-Pearson introduced PCA technique which needs probabilistic justifications which don't require a distributional assumption. It is one of the front-running unsupervised methods which find the subspace to reduce the training data reconstruction error. There are many successful applications are using PCA like face recognition, visual tracking, clustering, hyperspectral data land cover classification and so on. The huge multidimensionality of hyperspectral data are transformed to Eigen-space vectors of PCA and it is computed to synthesize the dataset with less computational complexity. In [2] volumetric 3D visualization data is experimented by improving visual quality. The training labels are subdivided by using the following techniques: Band based PCA and it is extended with time-varying volume data compression directly.

In HS data, continuous variation of the pixels from band to band in an image can be found. Here PCA uses the statistical analysis to evaluate the correlation and dependency between bands. From the experimentation of AVIRIS and HYDICE, hyperspectral dataset concluded the first few components offers the minimum of $70 \%$ of the correct classification accuracy [4]. The HS in medical application is a thriving world widely; in [5] to distinguish the region of interest in cancer caring nude mouse PCA and K-Means algorithms are adapted to select the highly informative wavelength bands. 
The non-linear PCA (NLPCA) is extended by deriving the major properties of PCA which is performed by the auto-associative neural network. The extended morphological profiles are created using NLPCA shows better results than the conventional PCA method [6].

Target detection in HS data is front running application where PCA offers the minimal impact on many algorithms such as Kelly's GLRT, Matched Filter-MF, orthogonal subspace projection-OSP, subspace version GLRT, adaptive-MF, ACE, and CEM. The above seven algorithms are the adaptive detection which contains variance and subspace-based detectors [7]. In [8], for extended morphological profiles, PCA and ICA are employed to verify the highest accuracy. AVIRIS Indian pines HS data improves the accuracy by extending morphological profiles with PCA technique for effective dimensionality reduction.

In HS edge preserving filter application, the edge preserving features (EPF) are gained for the effectual categorization of spatial and spectral structures of the objects in a given scene. Due to edge-preserving smoothing operation there is an unavoidable reduction of spectral differences among the objects can occur which reduce the correct classification performance. PCA is combined with EPF (PCA-EPFs) overcome the above issue and the results accuracy achieved very high with Support Vector Machine (SVM) classification [9]. In [10], for the targeted brain surgery application of HS data PCA parallelization technique is evolved in minimizing the time for capturing the frame-per minute. The vector quantization and PCA technique are combined to de-correlate the process of spectral information to compress the HS image ineffective way [11]. The detection of fault or damage in structures the wind turbine will be identified using the PCA method. The steps involved in this proposed work are; 1) the new measures of the sensors are projected to the PCA baseline model 2) structure is often inspected or supervised [12]. The Blind source separation-BSS methods are used to unravel the signal processing issue and also it uses the set of accessible signals which are mixtures with limited knowledge; mixing transformation taken place also referred unsupervised unmixing methods. The framework obtained for the above problem by analyzing the spectral variability in a large dimensional data, BSS methods are extended using nonnegative matrix factorization techniques which helps in handling the variability in datasets of intra-class are done by inverting the PCA techniques to BSS framework. The proposed study proves that PCA is often increasing the performance of the HS data unmixing [13].

\section{B. Independent Component Analysis}

In general, the ICA technique is mostly used in hyperspectral unmixing issues. Based on two assumptions in HS unmixing ICA is formed: 1) In a linear spectral mixture of a particular pixel in the endmember spectra are identified and weighted by the respective fraction of the pure materials as abundances and 2) sources are statistically not dependant. ICA is derived from Independent factor Analysis (IFA) wherein ICA first assumption is valid but the second assumption is violated. The accuracy of HS unmixing data is depending on the variability of signature, the number of endmembers and signal-noise-ratio (SNR). It is concluded that ICA is extended from IFA due to noise in HS data [14].

The BSS is a spotted issue in HS image where the pure substances are mixed in each macro or micro pixel. The

unsupervised ICA technique involves without prior knowledge of the substances present in the pixel. The contextual ICA (eICA) is generalized for the unrestricted form of distributions to unmix the pure substances in the subpixel [15]. To unmix the pure substances from the subpixel ICA alone is not required to complete the task and performance. So, Bayesian positive-BP source separation (BPSS) method adapted as a pre-processing step and ICA is involved after that which offers the high accuracy in separating the pure substances [16]. To estimate the pure spectral substance and abundance fraction amount of spectral information are identified thorough ICA-aided technique is proposed. This method uses the apriori knowledge about the pure substance present in the mixed pixel called end members. The derivation flow for this work is: Initially, Mixed pixel HS data are gained from the field of agriculture. Apply the ICA technique by including the green peak as an input to HS data. Independent components are identified as a component and noise by normalized and zero mean respectively [17].

Extracting the information about the location of chemicals like $\mathrm{CO}_{2}$ and $\mathrm{H}_{2} \mathrm{O}$ ices from Mars planet, the proposed work initially uses the ICA to extract the endmembers. Here the Independent components are weak due to the independence assumption of the basic properties are not verified thoroughly. In order to improve the extraction of components the Spatial ICA and with spectral-BPSS are combined together to achieve the high performance of the HS data [18]. ICA mixture model (ICAMM) is introduced to solve the conventional problem of the unsupervised classification techniques which often adapts multivariate Gaussian distribution for each class of HS data. ICAMM algorithm considered about the model class distributions as non-Gaussian density which shows the significant results in classification accuracy when compared with PCA, segmented PCA (SPCA), OSP and projection pursuit (PP) algorithms [19].

\section{SUPER VISED FEATURE EXTRACTION TECHNIQUES WITH REFERENCE TO HS DATA}

The chapter offers an overview of the HS data feature extraction and insight analysis of the Discriminant analysis techniques and NWFE techniques in the forthcoming sections.

\section{A. Discriminant Analysis of HS data in feature extraction}

In HS image classification, the Fisher's-LDA utilize the given ratio of given pure substances by Between--class to a Within--class matrix to obtain the feature vectors from projecting high to low dimensionality which means of maximizing the class separability. By considering the optimality the fisher's ratio is replaced with Linear Constraint-based discriminant analysis (LCDA); based on the criterion the ratio of inter-distance of the vector to intra-distance [20]. In [21] for HS dimensionality reduction the sparsity-preserving graph construction method is adapted in particular, if the labels are known then sparsity-based-GDA are concentrated by forcing the projection in along the direction of clustered samples in the sense of with-in class samples are represented. In SGDA, 
individual samples are represented for each class in HS image. It lacks in the global structure of the data, to overcome the global issue of data a sparse and low-rank graph based-SLGDA is used. Here, the low-rank depiction concentrates on preserving data structures of global. SLGDA achieved high performance when compared with LDA and PCA [22].

Generalized discriminant analysis (GDA) is a non-linear discrimination by adapting eigenvalue algebraic formulation. This GDA method uses the kernel function as a choice for giving a precise solution for some points. In case of small database, GDA outperforms well when compared with SVM and probabilistic neural network classifier [23]. The non-parametric statistics technique, the penalized discriminant analysis (PDA) introduced to classify the HS image of Sierra Nevada mountains in California. Here, the geometric point of view is emphasized and substantially improved the PDA upon LDA [24]. Basically, the supervised FE technique emphasizes on two conditions for handling dimensionality which are between-class-scatter and within-class-scatter. The proposed method feature space discriminant analysis initiates the following steps: If concurrently between-class-scatter is maximized and within-class-scatter is minimized then the extracted features differences are increased [25]. In [26] orthogonal linear discriminant analysis technique is adapted to ensure both spatial and spectral domains of HS image features are extracted for classification. Here, the sets of orthogonal filter and the transformation of the spectral information from HS data are framed to gain the maximum separability of each class. SVM classification technique is adapted to justify other techniques upon proposed method. Salinas, Pavia university and Indian pines HS images are used for the experimental study. In [27], Fundamentally, NWFE is amended from the standard LDA by replacing the local means from class means for every sample point. The weight of the scatter matrices is estimated using Euclidean distances to local means from every sample points. NWFE outperforms well upon LDA, where it uses the local knowledge and overcome the rank deficiency.

\section{Proposed Methodology Framework}

The proposed methodology framework for the feature extraction technique for HS data is shown in Figure 1. Initially, HS data are preprocessed by removing noisy bands, next FE technique is adapted to reduce the multi-dimensionality of the bands in the HS data. Once, the bands' dimensionalities are reduced then by training samples or without samples, the classification technique is employed to classify the labels of HS data. Finally, for justifying the algorithms kappa, Overall accuracy (OA) and average accuracy are estimated.

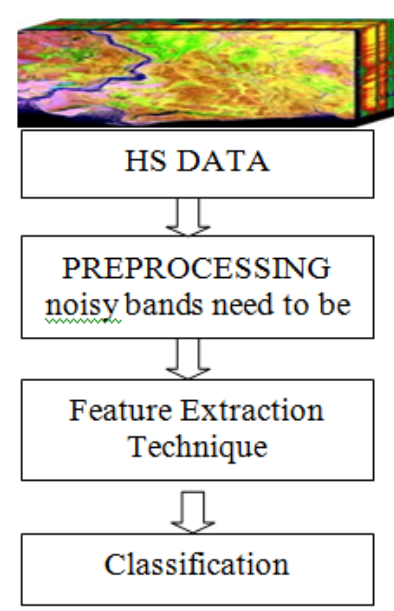

Figure 1. Generic methodology framework iv

\section{Proposed Work}

In this research work LDA, PCA, Prob PCA and GWMPCA techniques are employed and did the experimental results in a hyperspectral dataset. The proposed Gravitational mass formulation used for the weighted method in the unsupervised PCA is a novel fusion technique which is inspired by the optimization gravitational search algorithm (GSA)[28].

\section{A. Gravitational Mass Weighted (GMW)}

According to the theory of physics, the active gravitational mass (AGM), inertia mass (IM), and passive gravitational mass are the three different masses used to estimate the weights in different scenario [28]. Assume there are $N$ agents (bands); then the place of the spectral value $i$-th agent is defined in the following equation (1):

$$
G_{i}=\left(G_{i}^{1} \ldots \ldots G_{i}^{d} \ldots \ldots G_{i}^{n}\right), \quad \text { Where } i=1,2, \ldots \ldots N
$$

In GSA, the place of the object denotes the answer to the issue and mass used as a fitness function. Here $G_{i}{ }_{i}$ represents the place information $i^{\text {th }}$ spectral value in the $d$-th and $n$-th dimensionality. Fundamentally, the objects with gravitational force (GF) contain high mutual attraction. The global movement of all the objects lead by GF will have heavier mass. If the mass of the objects is heavier then it leads to high performance. We can identify the GF and heavier mass objects using fitness value. So the heavier mass signifies the more attractions and also a good solution. The GMW is formulated and updated as follows:

Where the fitness value $f_{i}(j)$ is the $i^{\text {th }}$ spectral value of estimated fitness value at the iteration $j$, and for a minimization problem: $W_{i}(j)$ and $B_{i}(j)$ are worst and best respectively are estimated using the following equations (2) and (3):

$$
\begin{aligned}
& W(j)=\max _{i \in 1,2, \ldots \ldots N} f_{i}(j) \\
& B(j)=\min _{i \in 1,2, \ldots \ldots N} f_{i}(j)
\end{aligned}
$$


For a maximization problem: $W_{i}(j)$ and $B_{i}(j)$ are estimated using the following equations (4) and (5):

$$
\begin{aligned}
& W(j)=\min _{i \in 1,2, \ldots . N} f_{i}(j) \\
& B(j)=\max _{i \in 1,2, \ldots . . N} f_{i}(j)
\end{aligned}
$$

After estimation of maximization and minimization of $W_{i}(j)$ and $B_{i}(j)$, the mass will be updated by following equations (6) and (7) respectively.

$$
\begin{gathered}
m_{i}(j)=\frac{f_{i}(j)-W(j)}{f_{i}(j)-B(j)} \\
M_{i}(j)=\frac{m_{i}(j)}{\sum_{k}^{N} m_{k}(j)}
\end{gathered}
$$

The average $M_{i}(j)$ is considered as the weighted value. In this work, the multimodal function equation (8) used as an objective function to calculate the fitness of each agent $i$ :

$$
f_{i}(j)=\sum_{i=1}^{N}-G_{i} \sin \left(\sqrt{\left|G_{i}\right|}\right)
$$

There are different fitness functions are available such

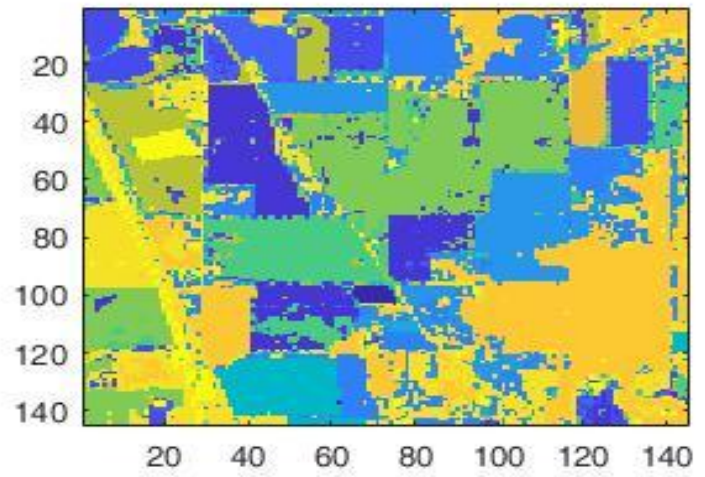

unimodal, multimodal and multimodal test functions with fix dimensions are available in the paper [28].

\section{B. Implementation of GMWPCA}

Algorithm 1 shows the proposed GMWPCA method: an unsupervised feature extraction PCA technique by adopting new GWM method.

\section{Results AND Discussion}

This research work got extended from the literature study by analyzing the existing techniques through the implementation in AVIRIS Indian pines dataset which consists of 220 bands with $145 \times 145 \times 220$. The Indian pines corrected bands are 200 and the remaining are removed which are noisy. This research work employed unsupervised PCA supervised LDA and GMWPCA techniques. The results are shown in Table 1 . There are total of 16 classes in AVIRIS Indian pines dataset with different samples present in the ground truth scene which is shown in Figure 2. The SVM classification technique is used to justify the feature extraction techniques. The overall accuracy achieved for PCA, probability PCA, LDA and GMWPCA are $88.97 \%$, $90.79 \%, 89.21 \%$, and $92.19 \%$ respectively. Figure 3, 4,5 and 6 shows the classification results of HS dataset.

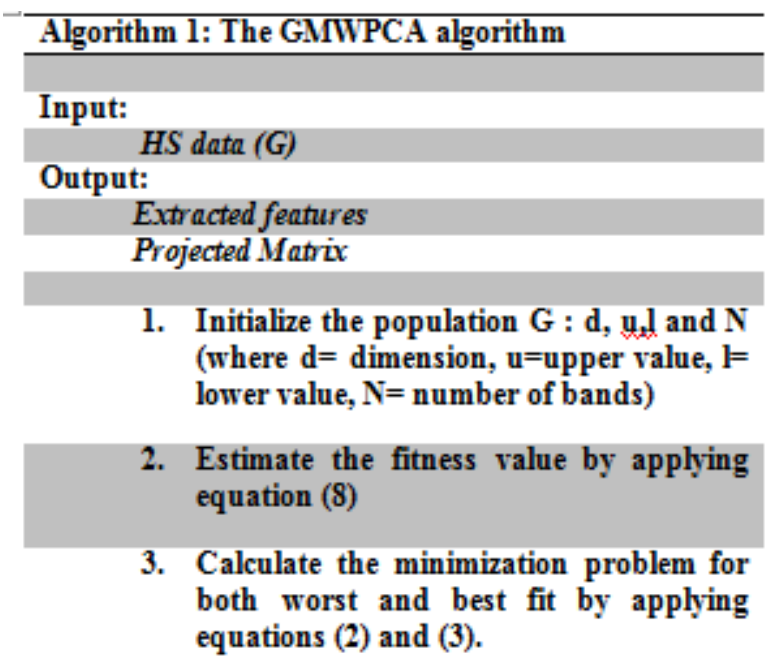

4. Calculate the maximization problem for both worst and best fit by applying equations (4) and (5)

5. Update the mass by applying equations (6) and (7)

6. The average of the mass $M_{i}(\mathcal{D})$ is considered as a weight value

7. Transform the matrix $\mathrm{G}$

8. Calculate the covariance matrix

9. Calculate the eigenvectors of the covariance matrix with GMW applied

10. Extract the features

11. Project the data into the matrix

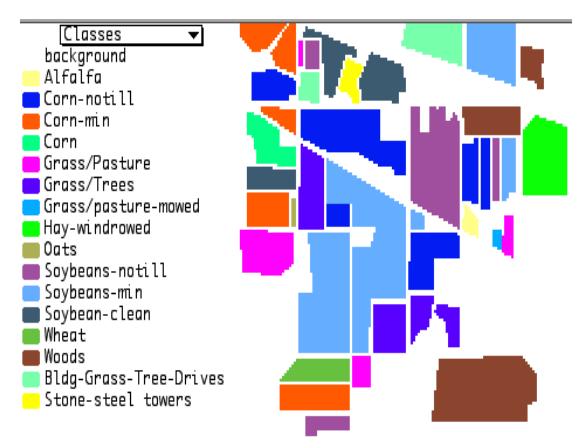

Figure 2. Ground truth information of AVIRIS Indian Pine

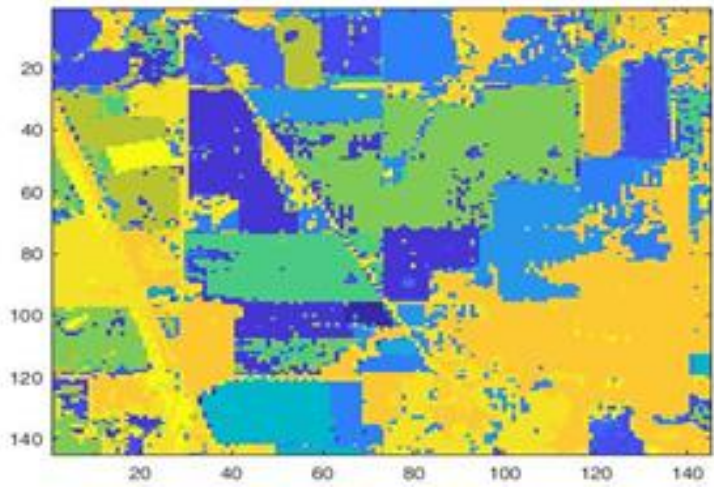

Figure 3. Results for PCA 


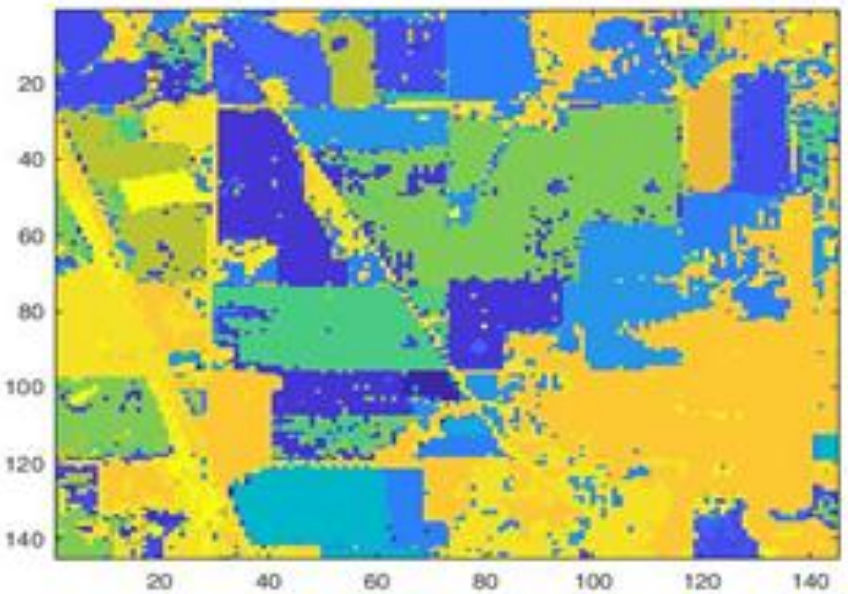

Figure 4. Results for Prob PCA

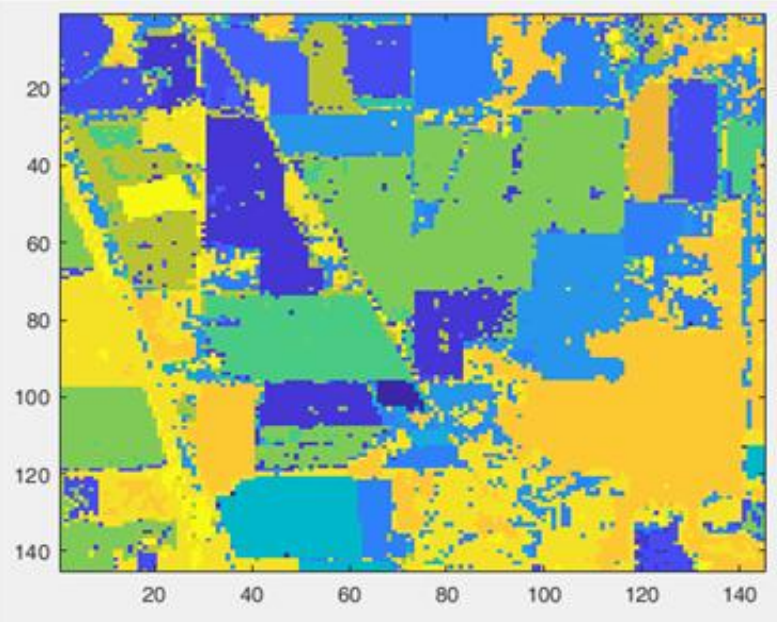

Figure 5. Results for LDA

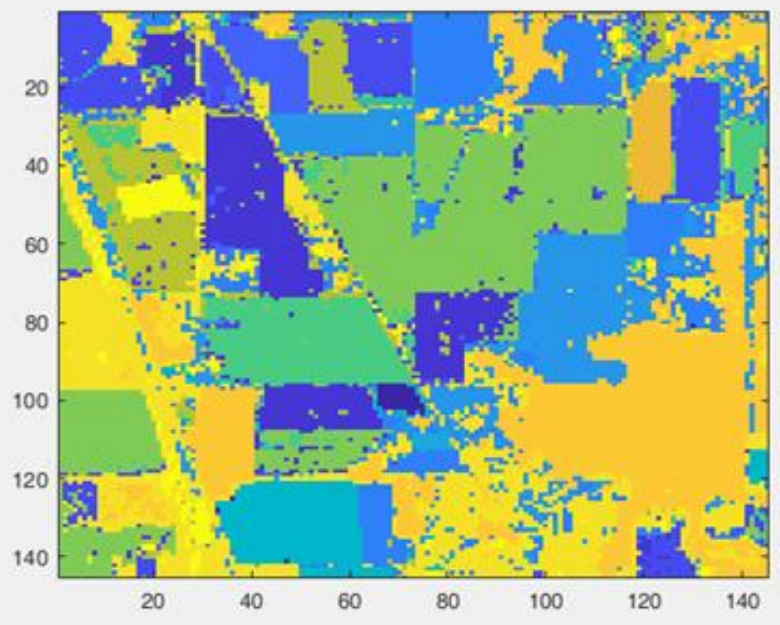

Figure 6. Results for GMWPCA

\begin{tabular}{|c|c|c|c|c|}
\hline \multirow{2}{*}{ Classes } & \multirow{2}{*}{$\begin{array}{l}\text { Supervised } \\
\text { LDA }\end{array}$} & \multicolumn{2}{|c|}{ Unsupervised } & \multirow{2}{*}{\begin{tabular}{|l|}
$\begin{array}{l}\text { Proposed } \\
\text { Method }\end{array}$ \\
GWMPCA
\end{tabular}} \\
\hline & & PCA & ProbPCA & \\
\hline 1 & $84.09 \%$ & $93.18 \%$ & $79.55 \%$ & $90.91 \%$ \\
\hline 2 & $82.01 \%$ & $84.68 \%$ & $85.98 \%$ & $84.86 \%$ \\
\hline 3 & $91.33 \%$ & $89.03 \%$ & $88.14 \%$ & $91.47 \%$ \\
\hline 4 & $94.02 \%$ & $88.59 \%$ & $93.48 \%$ & $92.53 \%$ \\
\hline 5 & $89.93 \%$ & $95.97 \%$ & $93.06 \%$ & $94.28 \%$ \\
\hline 6 & $97.70 \%$ & $96.56 \%$ & $97.56 \%$ & $98.40 \%$ \\
\hline 7 & $81.25 \%$ & $87.50 \%$ & $87.50 \%$ & $87.50 \%$ \\
\hline 8 & $99.32 \%$ & $98.63 \%$ & $98.63 \%$ & $99.07 \%$ \\
\hline 9 & $100.00 \%$ & $100.00 \%$ & $100.00 \%$ & $100.00 \%$ \\
\hline 10 & $88.24 \%$ & $84.53 \%$ & $83.66 \%$ & $88.22 \%$ \\
\hline 11 & $83.87 \%$ & $82.05 \%$ & $88.88 \%$ & $93.60 \%$ \\
\hline 12 & $90.60 \%$ & $89.18 \%$ & $90.96 \%$ & $89.35 \%$ \\
\hline 13 & $98.77 \%$ & $97.53 \%$ & $99.38 \%$ & $98.68 \%$ \\
\hline 14 & $94.61 \%$ & $96.70 \%$ & $96.30 \%$ & $93.84 \%$ \\
\hline 15 & $93.03 \%$ & $96.36 \%$ & $96.67 \%$ & $95.31 \%$ \\
\hline 16 & $100.00 \%$ & $95.56 \%$ & $100.00 \%$ & $100.00 \%$ \\
\hline Average & $91.80 \%$ & $92.25 \%$ & $92.48 \%$ & $93.63 \%$ \\
\hline OA & $89.21 \%$ & $88.97 \%$ & $90.79 \%$ & $92.19 \%$ \\
\hline Kappa & $87.70 \%$ & $87.43 \%$ & $89.46 \%$ & $91.04 \%$ \\
\hline
\end{tabular}

\section{CONCLUSION AND Future wORK}

From the above study and analysis identified that to improve the performance of the classification by adapting unsupervised feature extraction PCA techniques are often used by extending or modify the weighted techniques. In most of the HS feature extraction AVIRIS Indian pines HS data and Pavia university scene HS dataset are experimented often. In supervised feature extraction, GDA and NWFE are extended from LDA techniques. PDA, LDA, GDA, NWFE are widely used techniques. The overall background study concludes that, because of the constricted quantity of training labels present in HS data; the unsupervised techniques are used widely. From the experimental analysis identified that the proposed GMWPCA achieved high performance than the other existing algorithms such as PCA, probPCA and LDA. In future, the proposed method will be analyzed in a classification of sub-pixel.

\section{ACKNOWLEDGMENT}

This research study is supported under the University Research Fellowship (URF NO: C2/2016/2170) from the Bharathiar University, Coimbatore, India..

\section{REFERENCES}

1. Imani, Maryam, and Hassan Ghassemian. "Binary coding based feature extraction in remote sensing high dimensional data." Information Sciences 342 (2016): 191-208.

2. Alakkari, Salaheddin, and John Dingliana. "Principal Component Analysis Techniques for Visualization of Volumetric Data." Advances in Principal Component Analysis. Springer, Singapore, 2018. 99-120.

3. "Principal Component Analysis", http://kiwi.bridgeport.edu/cpeg540/PrincipalComponentAnalysis_Tut orial.pdf.

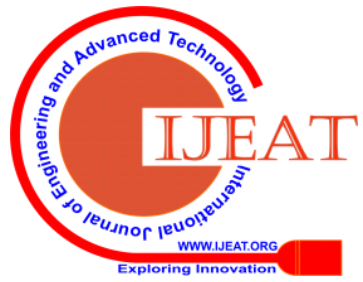


4. Rodarmel, Craig, and Jie Shan. "Principal component analysis for hyperspectral image classification." Surveying and Land Information Science 62.2 (2002): 115-122.

5. Kasai, Mai. "In Vivo Tumor Spatial Classification using PCA and K-Means with NIR-Hyperspectral Data." Journal of Biomedical Engineering and Medical Imaging 3.1 (2016): 45

6. Farrell, Michael D., and Russell M. Mersereau. "On the impact of PCA dimension reduction for hyperspectral detection of difficult targets." IEEE Geoscience and Remote Sensing Letters 2.2 (2005): 192-195.

7. Licciardi, Giorgio, et al. "Linear versus nonlinear PCA for the classification of hyperspectral data based on the extended morphological profiles." IEEE Geoscience and Remote Sensing Letters 9.3 (2012): 447-451.

8. Ghamisi, Pedram, et al. "Hyperspectral data classification using extended extinction profiles." IEEE Geoscience and Remote Sensing Letters 13.11 (2016): 1641-1645.

9. Kang, Xudong, et al. "PCA-based edge-preserving features for hyperspectral image classification." IEEE Transactions on Geoscience and Remote Sensing 55.12 (2017): 7140-7151.

10. Lazcano, Raquel, et al. "Porting a PCA-based hyperspectral image dimensionality reduction algorithm for brain cancer detection on a many-core architecture."Journal of Systems Architecture 77 (2017): 101-111.

11. Báscones, Daniel, Carlos González, and Daniel Mozos. "Hyperspectral Image Compression Using Vector Quantization, PCA and JPEG2000." Remote Sensing 10.6 (2018): 907.

12. Pozo, Francesc, and Yolanda Vidal. "Damage and fault detection of structures using principal component analysis and hypothesis testing." Advances in Principal Component Analysis. Springer, Singapore, 2018. 137-191.

13. Deville, Yannick, et al. "Application and Extension of PCA Concepts to Blind Unmixing of Hyperspectral Data with Intra-class Variability." Advances in Principal Component Analysis. Springer, Singapore, 2018. 225-252.

14. Nascimento, José MP, and Jose MB Dias. "Does independent component analysis play a role in unmixing hyperspectral data?." IEEE Transactions on Geoscience and Remote Sensing 43.1 (2005): 175-187.

15. Bayliss, Jessica D., J. Anthony Gualtieri, and Robert F. Cromp. "Analyzing hyperspectral data with independent component analysis." 26th AIPR Workshop: Exploiting New Image Sources and Sensors. Vol. 3240. International Society for Optics and Photonics, 1998.

16. Villa, Alberto, et al. "On the use of ICA for hyperspectral image analysis." Geoscience and Remote Sensing Symposium, 2009 IEEE International, IGARSS 2009. Vol. 4. IEEE, 2009.

17. Kosaka, Naoko, Kuniaki Uto, and Yukio Kosugi. "ICA-aided mixed-pixel analysis of hyperspectral data in agricultural land." IEEE Geoscience and Remote Sensing Letters 2.2 (2005): 220-224.

18. Moussaoui, Saïd, et al. "On the decomposition of Mars hyperspectral data by ICA and Bayesian positive source separation." Neurocomputing 71.10-12 (2008): 2194-2208.

19. Shah, Chintan A., Manoj K. Arora, and Pramod K. Varshney. "Unsupervised classification of hyperspectral data: an ICA mixture model based approach." International Journal of Remote Sensing 25.2 (2004): 481-487.

20. Du, Qian, and Chein-I. Chang. "A linear constrained distance-based discriminant analysis for hyperspectral image classification." Pattern Recognition 34.2 (2001): 361-373.

21. Ly, Nam Hoai, Qian Du, and James E. Fowler. "Sparse graph-based discriminant analysis for hyperspectral imagery." IEEE Transactions on Geoscience and Remote Sensing 52.7 (2014): 3872-3884.

22. Li, Wei, Jiabin Liu, and Qian Du. "Sparse and low-rank graph for discriminant analysis of hyperspectral imagery." IEEE Transactions on Geoscience and Remote Sensing 54.7 (2016): 4094-4105.

23. Baudat, Gaston, and Fatiha Anouar. "Generalized discriminant analysis using a kernel approach." Neural computation 12.10 (2000): 2385-2404.

24. Yu, Bin, et al. "Penalized discriminant analysis of in situ hyperspectral data for conifer species recognition." Ieee transactions on Geoscience and remote sensing 37.5 (1999): 2569-2577.

25. Imani, Maryam, and Hassan Ghassemian. "Feature space discriminant analysis for hyperspectral data feature reduction." ISPRS Journal of Photogrammetry and Remote Sensing 102 (2015): 1-13.

26. Shahdoosti, Hamid Reza, and Fardin Mirzapour. "Spectral-spatial feature extraction using orthogonal linear discriminant analysis for classification of hyperspectral data." European Journal of Remote Sensing 50.1 (2017): 111-124.
27. Li, Fan, et al. "Feature extraction for hyperspectral imagery via ensemble localized manifold learning." IEEE Geoscience and Remote Sensing Letters 12.12 (2015): 2486-2490.

28. Rashedi, Esmat, Hossein Nezamabadi-Pour, and Saeid Saryazdi. "GSA: a gravitational search algorithm." Information sciences 179.13 (2009): 2232-2248.

\section{AUTHORS PROFILE}

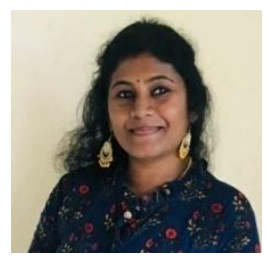

K. Nandhini, pursued MCA degree in school of distance education Bharathiar university, received M.Phil degree in Avinashilingam Deemed university and pursuing PhD in Bharathiar University. She awarded University research fellowship (URF) from Bharathiar university for research study in 2017, Her research interests include Text mining, Spatio-temoporal data mining, Visual Analytics and Hyperspectral remote sensing classification.

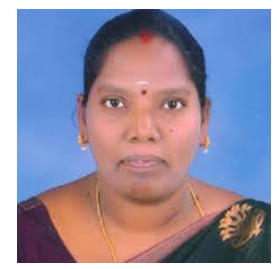

Dr. R. Porkodi, received MCA degree and pursued Ph.D in Bharathiar University. She received UGC grant for her research study. She is the member of many academic bodies. She is the life member in computer society in India and member in IAENG and IACSIT. She acted as a committee member/ resource person/coordinator for various research conferences/events/Faculty development programmes. She published many articles in various reputed journals. Her research interests include Data mining, NLP, Image mining, CBMIR, Hyperspectral remote sensing and Bioinformatics 